\title{
EXTREMAL VALUES OF CONTINUANTS
}

\author{
G. RAMHARTER
}

\begin{abstract}
The following question was posed by C. A. Nicol: Given an arbitrary set $B$ of positive integers, find the extremal denominators of regular continued fractions with partial denominators from $B$, each element occurring a given number of times. Partial solutions have been given by T. S. Motzkin and E. G. Straus, and later by T. W. Cusick. We derive the general solutions from a purely combinatorial theorem about the set of permutations of a vector with components from an arbitrary linearly ordered set. We also consider certain halfregular continued fractions. Here the maximizing arrangements have to be described in terms of an algorithmic procedure, as their combinatorial structure is exceptionally complicated. Its investigation leads to a connection with the well-known Markov spectrum. Finally we obtain an asymptotic formula for the ratio of extremal continuants and some sharp (essentially analytic) inequalities concerning cyclic continuants.
\end{abstract}

1. Introduction. For any set $B$ of positive integers let $E_{B}$ denote the set of (finite or infinite) regular continued fractions with partial denominators from $B$. There are several open problems connected with the sets $E_{B}$. They have Lebesgue measure 0 (for $B \neq \mathbf{N}$ ), and so their fractional (Hausdorff-) dimensions have attracted some interest (see e.g. V. Jarnik [5], I. J. Good [6], C. A. Rogers [13], I. Borosh [1], R. Kaufman [8]). The determination of the dimensional numbers is related to the distribution of continuants (i.e. denominators of continued fractions) with digits from $B$. For finite $B$ a remarkable connection with the enumeration of continuants was discovered by T. W. Cusick [3]. The result can be extended to arbitrary infinite $B$, and for its effective application (see [12]) it seemed desirable to solve the problem (posed by C. A. Nicol) of determining the extremal values of all continuants with digits from $B$, each digit occurring a given number of times. From a question in diophantine approximation [11] the analogous problem arose for halfregular continued fractions (cf. (1.2) below). T. S. Motzkin and E. G. Straus [10] settled the problem for regular continuants with pairwise different entries by a combinatorial argument. Cusick [3] found the maximizing arrangement for an arbitrary sequence of one's and two's.

In this paper we present the solutions for the general cases of both problems, any set $B$ of digits and any multiplicities being admitted (Theorem 1). We refer to the regular maximizing and minimizing arrangements as $X_{\max }, X_{\min }$ in the regular and

Received by the editors July 27, 1982.

1980 Mathematics Subject Classification. Primary 10A32; Secondary 05A20, 06A10.

Key words and phrases. Regular continued fractions, halfregular continued fractions, partial orderings, finite permutation groups, diophantine approximation, Markov spectrum, cyclic continuants, combinatorial inequalities, analytic inequalities. 
$X_{\max }^{\cdot}, X_{\min }^{\cdot}$ in the halfregular version. $X_{\max }$ and $X_{\min }^{\cdot}$ are of a similar combinatorial type and coincide if no repetitions occur; but surprisingly it is $X_{\min }^{\cdot}$ which appears as a direct generalisation of $X_{\max }$ as described in [10]. $X_{\min }$ has also a simple and permanent structure (generalising the respective result in [10]), much in contrast to an abundant variety of essentially different patterns for $X_{\max }^{\cdot}$. Here one has to be content with an algorithmic procedure which leads to the solution in a reasonable number of steps (in the case of two digits with arbitrary multiplicities, for instance,

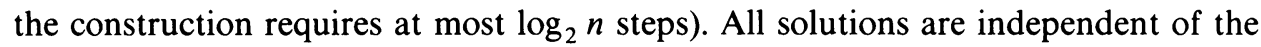
actual choice of $B$. In fact the proofs are based on a purely combinatorial theorem on the set $\mathfrak{X}$ of permutations of a vector with components from an arbitrary linearly ordered set $B$. It has become clear that the underlying problem is not primarily the extremisation of a real function, such as continuants, but $X_{\max }, X_{\min }$ (resp. $X_{\max }^{*}$, $X_{\min }^{\cdot}$ ) are proved to be themselves unique extremal elements of $\mathfrak{X}$ with respect to a natural semiordering generated by the linear ordering on $B$ (Theorem 2 ). In light of this, the asserted extremum property is an immediate consequence of the fact that (for integer sets $B$ ) continuants provide an order-preserving mapping of $\mathfrak{X}$ to the real axis (Theorem 3).

In view of the application mentioned we study the ratio of the extremal values for continuants of large order (Corollary 1) and obtain some sharp inequalities for cyclic continuants (Theorem 4; cf. [10,p. 1018 ff.]). In the last section we state a more general problem which is suggested by Theorem 1 .

2. Definitions and results. For rational $x \in(0,1)$ we consider the regular and halfregular expansions

$$
x=\frac{1}{x_{1}}+\frac{1}{x_{2}}+\cdots \frac{1}{x_{n}}=:\left[x_{1} \cdots x_{n}\right]
$$

and

$$
x=\frac{1}{x_{1}}-\frac{1}{x_{2}}-\cdots \frac{1}{x_{n}}=:\left[x_{1} \cdots x_{n}\right]
$$

with $x_{i} \in \mathbf{N}\left(x_{i} \geqslant 2\right.$ in the halfregular case). By $Q\left(x_{1} \cdots x_{n}\right)$ we denote the continuant of order $n$ with digits $x_{1}, \ldots, x_{n}$, i.e. the reduced denominator of a rational number $x$ with expansion (1.1). Similarly we use $Q^{\cdot}$ for (1.2). For the moment, let $B=\left\{b_{1}, \ldots, b_{r}\right\}$ be a fixed set of positive integers and assume $b_{1}<$ $\cdots<b_{r}$. We use the notation $(u)_{k},(U)_{k}$ to indicate a string of $k$ equal elements $u$ or segments $U(k \in\{0,1,2, \ldots, \infty\})$.

THEOREM 1. For given $n \in \mathbf{N}$ and given partition $P=\left(p_{1}, \ldots, p_{r}\right), p_{1}+\cdots+p_{r}=$ $n$, let $X=\left(x_{1} \cdots x_{n}\right)$ vary over the permutations of $\left(a_{1} \cdots a_{n}\right):=\left(\left(b_{1}\right)_{p_{1}} \cdots\left(b_{r}\right)_{p_{r}}\right)$. Then $Q_{\min }^{\cdot}=\min Q^{\circ}(X)$ and $Q_{\max }=\max Q(X)$ are uniquely attained for

$$
\begin{gathered}
X_{\min }^{\cdot}=\left(a_{2[(n-1) / 2]+1} \cdots a_{5} a_{3} a_{1} a_{2} a_{4} \cdots a_{2[n / 2]}\right), \\
X_{\max }=\left(\ldots b_{5} B_{4} b_{3} B_{2} b_{1} B_{1} b_{2} B_{3} b_{4} B_{5} \ldots\right),
\end{gathered}
$$


where $B_{k}:=\left(b_{k}\right)_{p_{k}-1}(k=1, \ldots, r) . Q_{\min }$ is uniquely attained for $X_{\min }$ which is, in the cases $n=2 k, 4 k-1,4 k+1$, given by

$$
\left\{\begin{array}{l}
\left(a_{1} a_{2 k} a_{3} a_{2 k-2} \cdots ; \cdots a_{2 k-3} a_{4} a_{2 k-1} a_{2}\right), \\
\left(a_{1} a_{4 k-1} a_{3} a_{4 k-3} \cdots a_{2 k-1} a_{2 k+1} ; a_{2 k} a_{2 k+2} \cdots a_{4 k-4} a_{4} a_{4 k-2} a_{2}\right), \\
\left(a_{1} a_{4 k+1} a_{3} a_{4 k-1} \cdots a_{2 k+3} a_{2 k+1} ; a_{2 k+2} a_{2 k} \cdots a_{4 k-2} a_{4} a_{4 k} a_{2}\right) .
\end{array}\right.
$$

As regards $X_{\max }^{\cdot}$, there is an infinity of essentially different patterns. This is illustrated by the fact that the closure of the set $\Theta=\left\{x=x(P)=\left[X_{\max }^{\cdot}\right] \mid p_{1}, \ldots, p_{r} \in\right.$ $\{0,1,2, \ldots\}\}$ has cardinality $c$, but is denumerable for the other solutions, with obvious modifications for the sets considered. For $r=\operatorname{card} B=2, X_{\max }^{\cdot}(P)$ can be constructed by a division algorithm from $p_{1}, p_{2}$ in at most $\log _{2} n$ steps.

For our investigation of $\Theta$ we apply a construction borrowed from the theory of the Markov spectrum (see $[\mathbf{2}, \mathbf{4}, \mathbf{7}, \mathbf{9}]$ ). In fact the arguments can be modified and elaborated so as to yield a characterisation of the spectrum. We remark that, for $r>2, X_{\max }^{\cdot}$ can be obtained in less than $\left(\begin{array}{l}n \\ 2\end{array}\right)^{2}$ steps by use of our method (which is still better than $k \cdot n$ ! unsystematic trials).

From Theorem 1 we derive an asymptotic formula for the ratio of the extremal values realised by (3) and (4):

Corollary. Given $B$ as in Theorem 1 (card $B=r$ ), consider for all partitions $P=\left(p_{1}, \ldots, p_{r}\right)$ of $n \in \mathbf{N}$ the corresponding extremal values $Q_{\max }=Q_{\max }(P)$, etc. Then, for $n \rightarrow \infty$,

$$
\rho(P):=\log \left(Q_{\max }(P) / Q_{\min }(P)\right)^{1 / n} \text { and } \dot{\rho}(P):=\log \left(Q_{\max }^{\cdot}(P) / Q_{\min }^{\cdot}(P)\right)^{1 / n}
$$

remain bounded away from 0 iff $p_{k} / n$ remains bounded away from 1 for all $k \in$ $\{1, \ldots, r\}$.

Putting

$$
\sigma(P)=\frac{1}{n} \sum_{i=1}^{[n / 2]} \log \frac{\left[\left(a_{i} a_{n-i+1}+2\right)_{\infty}\right]}{\left[\left(a_{i}\right)_{\infty}\right]\left[\left(a_{n-i+1}\right)_{\infty}\right]},
$$

we have

$$
\rho(P)=\sigma(P)+O(1 / n) \quad(n \rightarrow \infty)
$$

Moreover,

$$
\sup _{B}\left\{\sup _{n, P} \sigma(P)\right\}=\frac{1}{2} \log \frac{1+\sqrt{5}}{2},
$$

the supremum taken over all sets $B$ with cardinalities $r \in \mathbf{N}$ and all possible partitions $P$.

As we want to put Theorem 1 in a more general context, let us for the following consider a set $B$ of $r$ objects $b_{k}$ with a linear ordering, $b_{1}<\cdots<b_{r}$, say. Fix any $n \in \mathbf{N}$, a partition $P=\left(p_{1}, \ldots, p_{r}\right), p_{1}+\cdots+p_{r}=n$, and a sequence $A=\left(a_{1}\right.$ $\cdots a_{n}$ ) with elements $a_{i} \in B$, each $b_{k}$ occurring $p_{k}$ times. In the set of permutations 
$X$ of $A$ we would like to identify each $X=\left(x_{1} \cdots x_{n}\right)$ with the reflected $X^{*}:=\left(x_{n}\right.$ $\left.\cdots x_{1}\right)$. The resulting set will be denoted by $\mathfrak{X}=\mathfrak{X}(B, P)$. We write $X \simeq Y$ if $X=Y$ or $X=Y^{*}$. For segments $U=\left(u_{1} \cdots u_{k}\right) \in B^{k}$ with arbitrary length $k \in \mathbf{N}$ we introduce a lexicographical ordering, writing

$$
\begin{aligned}
U=\left(u_{1} \cdots u_{k}\right) \lessdot V=\left(v_{1} \cdots v_{m}\right) \quad & \text { if } u_{i}=v_{i}(i=1, \ldots, j-1), \\
& \text { and } u_{j}<v_{j} \text { for some } j \in\{1,2, \ldots\},
\end{aligned}
$$

with the understanding $u_{k+1}=\cdots=u_{m}=\infty$ if $k<m$, and vice versa. In particular, we assign $(\infty)$ to the empty segment. Similarly we write

$$
\begin{aligned}
U<V \quad \text { if } u_{1}<v_{1} ; & \text { or } \quad u_{1}=v_{1}, u_{2}>v_{2} ; \\
& \text { or } \ldots u_{i}=v_{i}(i=1, \ldots, 2 j-1), u_{2 j}>v_{2 j} ; \ldots
\end{aligned}
$$

and so on in alternating order. For an arrangement

$$
\left(u_{k} \cdots u_{1} ; v_{1} \cdots v_{s} ; w_{1} \cdots v_{m}\right)=U^{*} V W=X,
$$

we call the segment

$$
\begin{array}{ll}
V \text { regular } & \text { (with respect to }<\text { ) if } V<V^{*}, U<W \text { or } V>V^{*}, U>W, \\
& \text { (with respect to } \lessdot \text { ) if } V \lessdot V^{*}, U \lessdot W \text { or } V>V^{*}, U \gg W,
\end{array}
$$

(that is if the "smaller" endpoint of $V$ is attached to the smaller of the neighboring segments), and irregular if the smaller endpoint is attached to the greater segment. Note that the properties (6) are indeed invariant under reflection of $X$. For any pair of elements $X, X^{\prime} \in \mathfrak{X}$ we write $X \succ X^{\prime}$ (resp. $X \prec X^{\prime}$ ) if $X^{\prime}$ can be obtained from $X$ by inverting a regular segment. We will later see that there is a canonical way to do this and both relations are antisymmetric. Segments which are neither regular nor irregular can be disregarded, as their inversion does not affect $X$ (up to reflection). Therefore such segments will occasionally be called uninvertible. Finally, to obtain the desired transitivity, we write $X \ll Y$ (resp. $X \ll Y)$ for $X, Y \in \mathfrak{X}$ if there exists a sequence $X \prec X^{\prime} \prec X^{\prime \prime} \prec \cdots \prec Y$ (resp. $X \prec X^{\prime} \prec \cdots \prec Y$ ) with $X^{\prime}, X^{\prime \prime}, \ldots \in \mathfrak{X}$. It should be noticed that for the definitions of $\ll$, « we used nothing but the ordering on $B$.

LEMMA. Both relations $\ll$ and $\ll$ define a semiordering (not the same) on $\mathfrak{X}(B, P)$.

Any endpoints of maximal chains in $\mathfrak{X}$ will be called extremal (maximal or minimal).

THEOREM 2. Under the present assumptions, $(\mathfrak{X}, \ll)$ (resp. $(\mathfrak{X}, \ll))$ have unique maximal and minimal elements $X_{\max }, X_{\min }$ (resp. $X_{\max }^{\cdot}, X_{\min }^{\cdot}$ ) with exactly the properties stated in Theorem 1.

In our original problem we had to consider an integer set $B$ and functions $Q, Q^{*}$ defined on $\mathfrak{X}(B, P)$. The applicability of Theorem 2 arises from the fact that inverting a regular segment of the sequence of digits in a continuant decreases $Q(X)$ (resp. increases $Q^{\cdot}(X)$ ): 
TheOREM 3. For an integer set $B$ (as in Theorem 1) and a partition $P$ of $n$ let $\mathfrak{X}=\mathfrak{X}(B, P)$ be endowed with the relations $\ll($ resp. $\ll)$ generated by the usual order relation on the reals. Then for $X, Y \in \mathfrak{X}$,

$$
X \ll Y \text { implies } Q(X)<Q(Y)
$$

and

$$
X \ll Y \text { implies } Q^{\cdot}(X)<Q^{\cdot}(Y) \text {. }
$$

In other words, the mappings $Q: \mathfrak{X} \rightarrow \mathbf{R}\left(Q^{\bullet}: \mathfrak{X} \rightarrow \mathbf{R}\right)$ take increasing chains in $\mathfrak{X}$ to increasing chains in $\mathbf{R}$. The assertions of Theorem 1 now follow from the uniqueness of the extremal elements in $\mathfrak{X}$.

3. Proof of the Lemma. Let $X, X^{\prime} \in \mathfrak{X}$ be given and suppose that $X^{\prime}$ can be obtained from $X$ by inverting a segment. By $\mathfrak{B}$ we denote the set of segments $V$ of $X$ whose inversion has the same effect (that is, for any $V, V_{1} \in \mathfrak{B}$ one has $X=U^{*} V W$ $=U_{1}^{*} V_{1} W_{1}$ and $X^{\prime}=U^{*} V^{*} W=U_{1}^{*} V_{1}^{*} W_{1}$ with some $\left.U, W, U_{1}, W_{1}\right)$. We claim that for $V, V_{1} \in \mathfrak{B}$ there are only the possibilities $V=V^{*}$ (and thus $V_{1}=V_{1}^{*}, X \simeq X^{\prime}$ ) or $V_{1}=S^{*} V S$ or $V=S^{*} V_{1} S$ with some segment $S$. It suffices to consider the cases: (i) $V$ contained in $V_{1}$; (ii) $V, V_{1}$ overlapping; (iii) $V, V_{1}$ disjoint. Let us look at (i). Here $V_{1}=S^{*} V S^{\prime}$ with some $S, S^{\prime}$. We may suppose that $S$ is not longer than $S^{\prime}$. Then $S^{\prime}=R T$ and $V_{1}=S^{*} V R T$ with $S, T$ having the same length $(S, R, T$ possibly empty). As inversions of $V, V_{1}$ are assumed to have the same effect, it follows that $S^{*} V^{*} R T=T^{*} R^{*} V^{*} S$, hence $S=T$ and $V^{*} R=R^{*} V^{*}$. It is readily verified that the latter implies $V=V^{*}$, except possibly if $R$ is empty. But then indeed $V_{1}=S^{*} V S$. Similarly we are left with $V=V^{*}$ for (ii). For (iii) this is trivial. We conclude that there is a unique $\bar{V} \in \mathfrak{B}$ (called canonical segment of the pair $X, X^{\prime}$ ) with the property that for any $V \in \mathfrak{B}, \neq \bar{V}$, one has $\bar{V}=S^{*} V S$ with some $S$. Besides, $\bar{V}$ has maximal length. As for the regularity we show that all the invertible segments $V \in \mathfrak{B}$ are of one kind (regular or irregular). Indeed for any nonsymmetric $V \in \mathfrak{B}$ we have $\bar{V}=S^{*} V S$ and $X=\tilde{U}^{*} \bar{V} \tilde{W}=(S \tilde{U})^{*} V(S \tilde{W})=U^{*} V W$, hence

$$
\bar{V}<\bar{V}^{*} \text { iff } S^{*} V S<S^{*} V^{*} S \text { and } \quad U<W \text { iff } S \tilde{U}<S \tilde{W} .
$$

The same equivalences hold for $\lessdot$. But from the properties of both order relations it follows at once that $\bar{V}$ is regular iff $V$ is regular. Consequently, $X \prec X^{\prime}\left(X \neq X^{\prime}\right)$ excludes $X \succ X^{\prime}$, and the same with $\prec$; in other words, the relations are antisymmetric. The transitivity and antisymmetry of $\ll$ and $\ll$ is obvious from the definition and the fact that there exist (various) order-preserving real valuations of $\mathfrak{X}$ (as, for example, those described by Theorem 3). This proves the Lemma.

4. Proof of Theorem 3. The considerations for the expansions (1.1), (1.2) are running parallel as far as their elementary theory does. The final difference of the results is explained by the fact that $[\cdot]$, unlike $[\cdot]^{\cdot}$, is alternatively decreasing and increasing with the partial denominators, in accordance with the properties of the orderings $<$ and $\lessdot$ introduced. We record some basic relations. We do not distinguish between continuants as sequences of digits and the values assigned to 
them but no confusion should arise. For $X=\left(x_{1} \cdots x_{n}\right)$ we have

$$
Q(X)=\operatorname{Per} \tilde{X}, \quad Q \cdot(X)=\operatorname{Det} \tilde{X} \quad \text { with } \tilde{X}=\left(\begin{array}{ccccc}
x_{1} & 1 & & & \\
1 & x_{2} & \ddots & 0 & \\
& & \ddots & & \\
& 0 & \ddots & & 1 \\
& & & 1 & x_{n}
\end{array}\right) \text {, }
$$

where Per means the permanent of a matrix. To simplify the notation, we put $X_{s t}=Q\left(x_{s} \cdots x_{t}\right)$ for $s \leqslant t$ and use the conventions $X_{s s-1}=Q(\{\})=1,[\{\}]=0$ $\left(\{\}=\right.$ empty set). Similarly with $X_{s t}^{*}$. One has

$$
\begin{array}{cl}
Q(X)=Q\left(X^{*}\right), & Q^{\cdot}(X)=Q^{\cdot}\left(X^{*}\right), \\
X_{1 n}=X_{1 k} X_{k+1 n}+X_{1 k-1} X_{k+2 n}, & X_{1 n}^{*}=X_{1 k}^{*} X_{k+1 n}^{*}-X_{1 k-1}^{*} X_{k+2 n}^{*} \\
{[X]=X_{2 n} / X_{1 n}, \quad[X]^{*}=X_{2 n}^{*} / X_{1 n}^{*} .}
\end{array}
$$

Expanding $Q^{\cdot}(X)$ by iterated use of (9.2), one is led to a polynomial which is - apart from alternating signs-identical with the Euler polynomial $Q(X)$, i.e. the sum of all even-gapped subproducts of the digits $x_{1}, \ldots, x_{n}$. We are now ready to establish the announced properties (7). Application of (9.1)-(9.3) gives

$$
\begin{aligned}
Q\left(U^{*} V W\right) & =U_{1 k} V_{1 s} W_{1 m}+U_{2 k} V_{2 s-1} W_{2 m}+U_{2 k} V_{2 s} W_{1 m}+U_{1 k} V_{1 s-1} W_{2 m} \\
& =U_{1 k} W_{1 m}\left\{V_{1 s}+[U][W] V_{2 s-1}+\left([U] V_{2 s}+[W] V_{1 s-1}\right)\right\} ;
\end{aligned}
$$

hence

$$
\begin{aligned}
Q\left(U^{*} V W\right)-Q\left(U^{*} V^{*} W\right) & =U_{1 k} W_{1 m}\left(V_{2 s}-V_{1 s-1}\right)([U]-[W]) \\
& =U_{1 k} V_{1 s} W_{1 m}\left([V]-\left[V^{*}\right]\right)([U]-[W]) .
\end{aligned}
$$

Similarly,

$$
Q^{\cdot}\left(U^{*} V^{*} W\right)-Q^{\cdot}\left(U^{*} V W\right)=U_{1 k}^{\cdot} V_{1 s}^{\cdot} W_{1 m}^{\cdot}\left([V]^{\bullet}-\left[V^{*}\right]^{\bullet}\right)\left([U]^{\bullet}-[W]^{\bullet}\right) .
$$

But $[U]>[W]\left([U]^{\bullet}>[W]^{\circ}\right)$ iff $U<W(U \lessdot W)$. Thus inverting a regular segment decreases $Q$ and increases $Q^{\circ}$, which proves Theorem 3 .

Evidently the digits $b_{k}$ need not be integers, except for the occurrence of 2's in the halfregular case, where the last step of the proof might fail. Anyway the $b_{k}$ 's must have certain minimal distances. Generally (7) cannot hold everywhere for a continuous function on $\mathfrak{X}$, as Theorem 1 clearly shows.

5. Proof of Theorem 2 and a connection with the Markov spectrum. Let a linearly ordered set $B$ of objects $b_{k}\left(b_{1}<\cdots<b_{r}\right)$ be given. Fix $n \in \mathbf{N}$ and a partition $P=\left(p_{1}, \ldots, p_{r}\right), p_{1}+\cdots+p_{r}=n$. Accordingly define $\mathfrak{X}(B, P)$ and the semiorderings $\ll, \ll$. We begin with $X_{\max }, X_{\text {min }}^{\cdot}$, as they are treated similarly. Starting from any $X \in \mathfrak{X}$ and merely inverting segments with $\left(u_{1}-w_{1}\right)\left(v_{1}-v_{s}\right)<0$-which are certainly irregular (cf. (6)) - we move along a chain $X \prec X_{1} \prec X_{2} \prec \cdots \prec Y$ (resp. $\left.X>X_{1}>X_{2}>\cdots \succ Y\right)$, and necessarily end with an arrangement of the form

$$
Y\left\{s_{2}, \ldots, s_{r}\right\}=\left(\left(b_{r}\right)_{s_{r}} \cdots\left(b_{2}\right)_{s_{2}}\left(b_{1}\right)_{p_{1}}\left(b_{2}\right)_{t_{2}} \cdots\left(b_{r}\right)_{t_{r}}\right)
$$


with $s_{k} \in\left\{0, \ldots, p_{k}\right\}, s_{k}+t_{k}=p_{k}, k=2, \ldots, r$. By $\mathfrak{V}(\subset \mathfrak{X})$ we denote the set of elements (10.1). Put $\sigma_{k}=\left[p_{k} / 2\right], k=2, \ldots, r$, and let $h<l<m<q<\cdots$ be the indices with $p_{h}, p_{l}, \ldots$ odd. It is easily seen that all invertible segments of

$$
Y^{\cdot}=Y\left\{\sigma_{2}, \ldots ; \sigma_{h} ; \sigma_{h+1}, \ldots, \sigma_{l-1} ; \sigma_{l}+1 ; \sigma_{l+1}, \ldots ; \sigma_{m} ; \sigma_{m+1}, \ldots ; \sigma_{q}+1 ; \ldots\right\} \in \mathfrak{Y}
$$

are regular with respect to $\lessdot$. This characterises $Y^{\bullet}$ as a minimal element of $(\mathfrak{X}, \ll)$. It remains to prove the uniqueness. To this end, take any $Y \in \mathfrak{Y}$. If $\delta=s_{k}-t_{k} \geqslant 2$ (resp. $\delta \leqslant-2$ ) for a $k \in\{2, \ldots, r\}$, then the segment $V=\left(\left(b_{k}\right)_{\mu}\left(b_{k-1}\right)_{s_{k-1}}\right.$ $\left.\cdots\left(b_{k-1}\right)_{t_{k-1}}\left(b_{k}\right)_{\nu}\right)$ with $\mu=[|\delta| / 2], \nu=0$ (resp. $\mu, \nu$ interchanged) is certainly irregular with respect to $\lessdot$; for the neighbouring segment attached to the smaller endpoint is greater, because it has the shorter string of $b_{k}$ 's; and the next elements following in both directions are greater than $b_{k}$ (possibly $\infty$ ). Inversion of $V$ gives $Y^{\prime}=\left(\cdots\left(b_{k}\right)_{s_{k}} \cdots\left(b_{k}\right)_{t_{k}} \cdots\right) \prec Y$ with $\left|s_{k}-t_{k}\right| \leqslant 1$. This can be done for all $k=2, \ldots, r$, the argument being independent of the other lengths $s_{j}, t_{j}(j \neq k)$. We end with an arrangement $\tilde{Y}=Y\left\{s_{2}, \ldots, s_{r}\right\}$ for which $s_{k}=t_{k}=p_{k} / 2$ ( $p_{k}$ even) or $s_{k}=\left(p_{k} \pm 1\right) / 2\left(p_{k}\right.$ odd $)$. If now $\tilde{Y} \simeq Y^{*}$, we are finished. Otherwise, $\tilde{Y}$ can be adapted to $Y^{*}$ by inverting irregular segments as follows: Assume w.l.o.g. that $s_{h}=t_{h}-1$ for the smallest index with $p_{h}$ odd. If $s_{l}=t_{l}-1$ for the next index $l>h$ with $p_{l}$ odd, invert the segment

$$
V=\left(\left(b_{l}\right)_{s_{l}} \cdots\left(b_{h}\right)_{s_{h}} \cdots\left(b_{1}\right)_{p_{1}} \cdots\left(b_{h}\right)_{s_{h}+1} \cdots\left(b_{l}\right)_{s_{l}}\right),
$$

which is certainly irregular, no matter how the outer segments are placed. If $s_{l}=t_{l}+1$, let $V$ be unchanged. Proceeding successively like this, one ends with $Y^{\circ}$. Summarizing, we have seen that for any $X \in \mathfrak{X}$ there is a decreasing chain $X>$ $\cdots>Y^{*}$. This proves that there can be no other minimal element in $(\mathfrak{X}, \ll)$. But $Y^{\cdot}=X_{\min }^{\cdot}$ may be written in the form (2). By essentially the same reasoning, using the definition of $<$, it is shown that all invertible segments of

$$
\bar{Y}=Y\left\{p_{2}-1,1, p_{3}-1,1, p_{4}-1, \ldots\right\}
$$

are regular with respect to $<$ (which is the maximality property for $\ll$ ), and that any $Y \in \mathfrak{Y}$ can be adapted to $\bar{Y}$ by inverting irregular segments. Consequently, $\bar{Y} \gg X$ for all $X \in \mathfrak{X}$, hence $X_{\max }=\bar{Y}$, which proves (3). We proceed similarly for $X_{\min }$. Starting from any $X$ and inverting regular segments, one can at first produce an arrangement of the form

$$
\left(\left(b_{1} b_{r}\right)_{s_{1}}\left(b_{k_{2}} b_{j_{2}}\right)_{s_{2}} \cdots\left(b_{j_{t}}\right)_{s_{\tau}} \cdots\left(b_{j_{2}} b_{k_{2}}\right)_{t_{2}}\left(b_{r} b_{1}\right)_{t_{1}}\right)
$$

with $s_{i}+t_{i}=m_{i}, t_{i}, s_{i} \in\{0,1, \ldots\}(i=1, \ldots, \tau-1)$, where $\tau, m_{i}, b_{1} \leqslant b_{k_{2}} \leqslant b_{k_{3}} \leqslant$ $\cdots$, and $b_{r} \geqslant b_{j_{2}} \geqslant \cdots$ are taken in an obvious way as they occur in (4). But by appeal to the properties of the order relation $<$ on the segments, it is again easily seen that (4) has the minimality property (all invertible segments irregular with respect to $<$ ). Moreover, the arrangement is accessible from any element (10.2) by successively inverting appropriate regular segments containing the central segment $\left(b_{j_{\tau}}\right)_{s_{\tau}}$. This proves that the unique minimal element of $(\mathfrak{X}, \ll)$ is indeed given by (4).

Next we determine $X_{\max }^{*}=: X(p, q)$ for sequences $X=\left(x_{1} \cdots x_{n}\right)$ with $x_{i} \in B=$ $\{a, b\}, a<b$, and fixed multiplicities $p, q(p+q=n)$. Let $\mathfrak{X}=\mathfrak{X}(B ; p, q)$ be 
endowed with $\ll$. Trivially $X(0, q)=\left((b)_{q}\right), X(p, 0)=\left((a)_{p}\right)$. So let $p, q \geqslant 1$ and take any $X \in \mathfrak{X}$. If $X$ has an endpoint $x_{1}=b$, and there is an $x_{i}=a(i \neq 1, n)$, invert the segment $\left(x_{1} \cdots x_{i}\right)$-which is certainly regular - to obtain $X^{\prime}(>X)$ with endpoint $a$. In particular, this shows $X(1, q)=\left(a(b)_{q}\right), X(2, q)=\left(a(b)_{q} a\right)$. So for the following assume $p \geqslant 3, q \geqslant 1$.

The idea is to distinguish a certain subset $\overline{\mathfrak{X}}$ of $\mathfrak{X}$ which can be proved to contain all the maximal elements of $\mathfrak{X}$, and to establish an order-preserving bijective mapping $\overline{\mathfrak{X}} \leftrightarrow \mathfrak{X}\left(B^{(1)} ; p^{(1)}, q^{(1)}\right)$ with $B^{(1)}=\{0,1\}$ and some $p^{(1)}, q^{(1)}$ such that $n^{(1)}=p^{(1)}+q^{(1)}$ is smaller than $n / 2$, by which the search for $X_{\max }^{\cdot}$ is reduced to the analogous problem in a lower dimension $n^{(1)}$. This process can be iterated.

Let $X=\left(x_{1} \cdots x_{n}\right)$. First endpoints can be made $a$ 's. This done, in any pattern $\cdots b(a)_{s} V(b)_{t} \cdots(s, t \geqslant 2, V$ empty or $V=(b \cdots a))$, the blocks $(a)_{s},(b)_{t}$ can be reduced to lengths 1 and $|t-s|+1$ ( $=1$ if $s=t$, so that both are dissolved) by successively inverting $a V b, a b V a b, \ldots$ etc. As all these segments are regular with respect to $\lessdot$, we produce an increasing chain $X \prec X^{\prime} \prec \cdots$ which ends with an element of the form

$$
\left(a(b)_{t_{1}} a(b)_{t_{2}} a \cdots a(b)_{t_{p-1}} a\right), \quad t_{j} \in \mathbf{N}, \quad \text { when } p \geqslant q+1,
$$

or

$$
\left((a)_{s_{1}} b(a)_{s_{2}} b \cdots b(a)_{s_{q+1}}\right), \quad s_{j} \in \mathbf{N}, \quad \text { when } p \leqslant q+1 .
$$

For $p=q+1,(11.1)$ and (11.2) coincide to $\left((a b)_{q} a\right)$ which is already $X_{\max }^{*}$.

In the case $p<q+1$ consider arrangements with blocks $(b)_{t,}\left(t_{j} \in \mathbf{N}\right)$ separated by single $a$ 's (like (11.1)). If two block lengths differ by more than 1 , that is, a pattern $\cdots a(b)_{t} V(b)_{t+1+i} a \cdots$ occurs $\left(t, i \in \mathbf{N}, V=(a)\right.$ or $\left.V=\left(a V^{\prime} a\right)\right)$, then invert $\left(V(b)_{[(i+1) / 2]}\right)$-which is regular-to obtain an element of the same type but with new lengths differing by at most 1 . By balancing out block lengths like this as long as there are more than two values for the lengths, one is led along an increasing chain to an element of the form

$$
\bar{X}=\left(\bar{x}_{1} \cdots \bar{x}_{n}\right)=\left(a(b)_{t_{1}} a(b)_{t_{2}} a \cdots a(b)_{t_{p-1}} a\right), \quad t_{j} \in\{t, t+1\},
$$

with at most two values $t, t+1$ for the block lengths, $t$ occurring $p^{(1)}$ times, say, and $t+1 q^{(1)}$ times. Necessarily,

$$
\begin{aligned}
\sum t_{j}=p^{(1)} t+q^{(1)}(t+1)= & q, \\
& p^{(1)}+q^{(1)}=p-1, \quad 0 \leqslant q^{(1)} \leqslant p-1,
\end{aligned}
$$

must hold. We can exclude $q^{(1)}=p-1\left(p^{(1)}=0\right)$, as then only $t+1$ would occur, which is covered by taking $q^{(1)}=0\left(p^{(1)}=p-1\right)$; inserting this in (12.2) indeed increases $t$ by 1 . So (12.2) reads

$$
q^{(1)} \equiv q \bmod (p-1), \quad p^{(1)}=(p-1)-q^{(1)}, \quad 0 \leqslant q^{(1)}<(p-1),
$$

which shows that $p^{(1)}, q^{(1)}$ are uniquely determined. Let $\overline{\mathfrak{X}}$ denote the set of all elements of the form (12.1). (By imposing the condition (12.2) we make sure that each of them will indeed occur under the present assumption $p<q+1$.) Remember that, for any $X \in \mathfrak{X}$, there is an $\bar{X} \in \overline{\mathfrak{X}}$ with $X \ll \bar{X}$. It follows that the maximal 
elements of $\mathfrak{X}$ can be found in $\overline{\mathfrak{X}}$ (it is even true that ascending chains cannot leave $\overline{\mathfrak{X}}$ once they have arrived there; but we need not make use of this). We observe that there is a one-to-one correspondence between $\overline{\mathfrak{X}}$ and the set of vectors $\left(t_{1} \cdots t_{p-1}\right)$ which indicates the possibilities of grouping the $b$-blocks. For optical reasons we prefer to consider the set $\mathfrak{X}^{(1)}$ of vectors with reduced components

$$
\left(x_{1}^{(1)} \cdots x_{p-1}^{(1)}\right)=\left(t_{1}-t \cdots t_{p-1}-t\right) .
$$

Now evidently $\mathfrak{X}^{(1)}=\mathfrak{X}\left(B^{(1)} ; p^{(1)}, q^{(1)}\right)$ with $B^{(1)}=\{0,1\}$ and lower dimension $n^{(1)}=p^{(1)}+q^{(1)}=p-1 \leqslant n / 2$. Again we use the symbol $\ll$ for the natural semiordering on $\mathfrak{X}^{(1)}$. By use of the canonical inversion it can be shown that our correspondence $\mathrm{S}: \mathfrak{X}^{(1)} \leftrightarrow \overline{\mathfrak{X}}$ is order-preserving in both directions, but for our purpose it suffices to know that $T \ll T^{\prime}\left(T, T^{\prime} \in \mathfrak{X}^{(1)}\right)$ implies $\mathrm{S} T \ll \mathrm{S} T^{\prime}$. Take any pair of elements $T \prec T^{\prime}$. Then $T=U^{*} V W, T^{\prime}=U^{*} V^{*} W$ with a regular segment $V=(\tau-t \cdots \sigma-t)$. By definition $\mathrm{S} T=\bar{U}^{*} \bar{V} \bar{W}$ with $\bar{V}=\left(a(b)_{\tau} a \cdots a(b)_{\sigma} a\right)$. From the properties of $\lessdot$ and the structure of the elements (12.1) it follows at once that $\bar{V}$ is also regular in $T^{\prime}$, hence $\bar{U}^{*} \bar{V} \bar{W} \prec \bar{U}^{*} \bar{V}^{*} \bar{W}$. Since clearly $\bar{U}^{*} \bar{V}^{*} \bar{W}=$ $\mathrm{S}\left(U^{*} V^{*} W\right)\left(=\mathrm{S} T^{\prime}\right)$, we conclude that $\mathrm{S} T \prec \mathrm{S} T^{\prime}$, which gives the asserted implication. Consequently, if $\mathfrak{X}^{(1)}$ can be proved to have a unique maximal element $X_{\max }^{(1)}$, then $S X_{\max }^{(1)}$ must be the unique maximal element of $\overline{\mathfrak{X}}$ (and hence of $\mathfrak{X}$ ). In other words, the uniqueness of $X_{\max }^{(1)}$ anticipated, we have reduced our problem to the analogous one in $\mathfrak{X}^{(1)}$.

Finally, for $p \geqslant q+2$, consider arrangements with blocks $(a)_{s}$, separated by single $b$ 's. Starting from (11.2), a perfectly analogous process of balancing out lengths of $a$-blocks ends with

$$
\bar{X}=\left(\bar{x}_{1} \cdots \bar{x}_{n}\right)=\left((a)_{s_{1}} b(a)_{s_{2}} b \cdots b(a)_{s_{q+1}}\right), \quad s_{j} \in\{s, s+1\},
$$

with $s+1$ (resp. $s$ ) occurring $p^{(1)}$ (resp. $\left.q^{(1)}\right)$ times, where the multiplicities are uniquely determined by

$$
p^{(1)} \equiv p \bmod (q+1), \quad p^{(1)}=(q+1)-q^{(1)}, \quad 0<p^{(1)} \leqslant(q+1) .
$$

Again the set $\overline{\mathfrak{X}}$ of elements (14.1) contains all maximal elements of $\mathfrak{X}$. Let $\mathfrak{X}^{(1)}$ be the set of corresponding vectors

$$
T=\mathrm{S}^{-1} X=\left(x_{1}^{(1)} \cdots x_{q+1}^{(1)}\right)=\left(1-\left(s_{1}-s\right) \cdots 1-\left(s_{q+1}-s\right)\right)
$$

(the 0 's and l's being interchanged now). Here $\mathfrak{X}^{(1)}=\mathfrak{X}\left(B^{(1)} ; p^{(1)}, q^{(1)}\right)$ with $B^{(1)}=$ $\{0,1\}$ and dimension $n^{(1)}=p^{(1)}+q^{(1)}=q+1 \leqslant n / 2$. Again $\mathrm{S} T \ll \mathrm{S} T^{\prime}$, whenever $T \ll T^{\prime}$, with the same consequences as before.

If now $p^{(1)} \leqslant 2$ or $q^{(1)}=0$ or $p^{(1)}=q^{(1)}+1$, we are done. Otherwise $p^{(2)}, q^{(2)}$ may be computed by use of the reduction formulas (12.3) or (14.2) and the problem is reduced to $\mathfrak{X}^{(2)}$, and so on. Clearly the algorithm ends with $p^{(m)} \leqslant 2$ or $q^{(m)}=0$ or $p^{(m)}=q^{(m)}+1$ for some $m$ with $2^{m} \leqslant n$. But then we have indeed a unique explicit solution $X_{\max }^{(m)}$ of the form $\left((1)_{\nu}\right)$ or $\left(0(1)_{\nu}\right)$ or $\left(0(1)_{\nu} 0\right)$ or $\left((01)_{\nu} 0\right)$. The pattern of $X_{\max }^{\cdot}$ can be reconstructed in at most $\log _{2} n$ steps by tracing back the algorithm. Besides, it has become clear that the structure of the solution depends on $p, q$ only (not on the choice of $a, b$ ). 
To complete the proof of Theorem 2, it remains to comment on the "limiting" combinatorial behaviour of the solutions. On inspection of (2)-(4) we find that in these cases the solutions are of the form $X(P)=\left(\left(A_{1}\right)_{k_{1}} \cdots\left(A_{m}\right)_{k_{m}}\right)$ with certain integers $k_{i}=k_{i}(P) \in\{0,1,2, \ldots\}$ and a fixed set of segments $A_{1}, \ldots, A_{m}$, independent of $P$ (indeed pairs $\left(b_{k} b_{j}\right),\left(b_{j} b_{k}\right)$ or single elements $b_{k}$, as can be seen from (10.1) and (10.2)). We let any integers $b_{1}^{\prime}<\cdots<b_{r}^{\prime}$ be assigned to the objects $b_{1}<\cdots<b_{r}$ and consider the corresponding solutions $X(P)$. (The final conclusions will not be touched by this choice. So the closure of $\Theta=\{[X(P)]\}$ (resp. $\left\{[X(P)]^{\circ}\right\}$ ) appears as a reasonable indicator of the combinatorial complexity of the solutions. One may even take $p$-adic or various other types of expansions instead of $[\cdot],[\cdot]^{*}$.) It is now evident that the accumulation points of $[X(P)]$ constitute a closed set of quadratic irrationalities (and eventually rationals for $[\cdot]^{\circ}$, as $\left[(2)_{\infty}\right]=1$ and there may occur arbitrarily long strings of 2's). Therefore clos $\Theta$ is denumerable.

For the remaining case it suffices to take card $B=2$, since clearly $\left\{X_{\max }^{\cdot}\left(p_{1}, \ldots, p_{r}\right)\right\} \supset\left\{X_{\max }^{\cdot}\left(p_{1}, p_{2}\right)\right\}$. Let $B=\{a, b\}, a, b \in \mathbf{N}, 2 \leqslant a<b$. Put $\mathfrak{M}=\left\{X_{\text {max }}^{\cdot}(p, q) \mid p, q \in \mathbf{N}\right\}, \Theta=\{[X] \mid X \in \mathfrak{M}\}$. We construct a certain subset $\mathfrak{Q}$ of $\mathfrak{M}$ which is closely connected with the well-known Markov spectrum and gives rise to continuously many accumulation points of $\Theta$. Let $U_{0}=(a), V_{0}=(b)$. We define inductively a set $\mathfrak{U}$ of pairs of segments as follows. $\left(U_{0}, V_{0}\right) \in \mathfrak{U}$. If $(U, V) \in \mathfrak{U}$, then $(U V, V) \in \mathfrak{U}$ and $(U, U V) \in \mathfrak{U}$. Here $U V$ means composition of segments. Let $\mathcal{L}^{\prime}$ be the set of segments $X^{\prime}$ occurring as a component of a pair $(U, V)$. To each $X^{\prime} \in \mathfrak{L}^{\prime}$ adjoin the smallest possible segment $S$ so as to make $X=X^{\prime} S$ symmetric. $S$ will certainly be shorter than $X^{\prime}$, because the two-sided infinite sequence $\cdots X^{\prime} X^{\prime} X^{\prime}$ ... (interpreted as a sequence of digits $a, b)$ is symmetric (i.e. a copy of its own reflection), which follows immediately from our construction. The set of elements $X$ obtained in this way is called $\mathfrak{R}$. By appeal to the algorithm in the preceding section, a simple inductive argument shows that each $X \in \mathfrak{L}$ is indeed $X_{\max }^{\cdot}(p, q)$ among all permutations with that partition. Now $\mathfrak{U}$ is a tree with continuously many different chains emanating from $\left(U_{0}, V_{0}\right)$. Accordingly there are continuously many chains $\left\{X_{1}^{\prime}, X_{2}^{\prime}, \ldots\right\}\left(X_{k}^{\prime}=U_{k} V_{k} \in \mathbb{R}^{\prime}\right.$; from any pair $(U, V)$ take the longer component). Obviously $\lim _{k \rightarrow \infty}\left[X_{k}^{\prime}\right]^{\cdot}$ (and so $\lim \left[X_{k}\right]^{\circ}$ ) exists and all these limits are different. Hence $\operatorname{clos} \Theta$ has cardinality $c$. The proof of Theorem 2 is complete.

6. Some facts about cyclic continuants. For the proof of Theorem 4 and the Corollary we need an identity for continuants with a period $A=\left(a_{1} \cdots a_{m}\right), m \geqslant 2$ ( $a_{i}$ not necessarily all distinct or arranged in monotonic order). Some calculation gives

$$
\begin{gathered}
Q^{\cdot}\left((A)_{p}\right)=Q^{\cdot}\left((\dot{q})_{p}\right)+Q^{\cdot}\left(A^{\prime}\right) Q^{\cdot}\left((\dot{q})_{p-1}\right), \\
\left\{\begin{array}{l}
Q\left((A)_{p}\right)=Q^{\cdot}\left((q)_{p}\right)-Q\left(A^{\prime}\right) Q^{\cdot}\left((q)_{p-1}\right) \quad(m \text { even }), \\
Q\left((A)_{p}\right)=Q\left((q)_{p}\right)-Q\left(A^{\prime}\right) Q\left((q)_{p-1}\right) \quad(m \text { odd }),
\end{array}\right.
\end{gathered}
$$

$p \in \mathbf{N}$, where $A^{\prime}=\left(a_{2} \cdots a_{m-1}\right)$ and

$$
\begin{aligned}
& q=q(A)=Q(A)+Q\left(A^{\prime}\right)=\operatorname{Per} \tilde{A}-2, \\
& \dot{q}=\dot{q}(A)=Q^{\cdot}(A)-Q^{\cdot}\left(A^{\prime}\right)=-\operatorname{Det} \tilde{A}-2(-1)^{m},
\end{aligned}
$$


with

$$
\tilde{A}=\left(\begin{array}{cccccc}
0 & 1 & 0 & \ldots & 0 & 1 \\
1 & a_{1} & 1 & & & 0 \\
0 & 1 & a_{2} & \ddots & & \vdots \\
\vdots & & \ddots & \ddots & 1 & 0 \\
0 & & & 1 & a_{m} & 1 \\
1 & 0 & \ldots & 0 & 1 & 0
\end{array}\right) .
$$

From the last representation it is evident that the nonconstant part of $q$ (resp. $\dot{q}$ ) is the (alternating) sum of all even-gapped subproducts of the digits $a_{1}, \ldots, a_{m}$, where these elements are regarded as cyclically ordered rather than linearly, and so $q, \dot{q}$ are just the cyclic continuants in the sense of [10, p. 1018].

For any integer $a$ we write

$$
\alpha=\llbracket a \rrbracket=1 /\left[(a)_{\infty}\right], \quad \dot{\alpha}=\llbracket a \rrbracket^{\cdot}=1 /\left[(a)_{\infty}\right]^{\cdot} .
$$

THEOREM 4. For cyclic continuants the inequalities

$$
\begin{gathered}
\llbracket \dot{q}\left(a_{1}, \ldots, a_{m}\right) \rrbracket^{\bullet} \geqslant \llbracket a_{1} \rrbracket^{\cdot} \cdots \llbracket a_{m} \rrbracket^{\cdot}, \\
\begin{cases}\llbracket q\left(a_{1}, \ldots, a_{m}\right) \rrbracket^{\cdot} \leqslant \llbracket a_{1} \rrbracket \cdots \llbracket a_{m} \rrbracket & (\text { m even }), \\
\llbracket q\left(a_{1}, \ldots, a_{m}\right) \rrbracket \leqslant \llbracket a_{1} \rrbracket \cdots \llbracket a_{m} \rrbracket & (\text { m odd }),\end{cases}
\end{gathered}
$$

hold with equality if the $a_{i}$ are all equal.

ProOF OF TheOREM 4. Using (9.2), one gets by induction:

$$
\begin{gathered}
Q \cdot\left((a)_{p-1}\right)=\left(\dot{\alpha}^{p}-\dot{\alpha}^{-p}\right) /\left(\dot{\alpha}-\dot{\alpha}^{-1}\right), \quad a>2, \quad Q \cdot\left((2)_{p-1}\right)=p, \\
Q\left((a)_{p-1}\right)=\left(\alpha^{p}-(-\alpha)^{-p}\right) /\left(\alpha+\alpha^{-1}\right)
\end{gathered}
$$

$(p \in \mathbf{N})$. For a sequence composed of segments $U_{1} U_{2} \cdots U_{s}$ the following trivial inequalities hold, again by (9.2):

$$
\begin{gathered}
Q\left(U_{1}\right) \cdots Q\left(U_{s}\right)<Q\left(U_{1} \cdots U_{s}\right)<C^{s-1} Q\left(U_{1}\right) \cdots Q\left(U_{s}\right), \\
Q^{\cdot}\left(U_{1}\right) \cdots Q^{\cdot}\left(U_{s}\right)>Q^{\cdot}\left(U_{1} \cdots U_{s}\right)>D^{s-1} Q^{\cdot}\left(U_{1}\right) \cdots Q^{\cdot}\left(U_{s}\right)
\end{gathered}
$$

with absolute constants $C, D$. For the right side of (19.2) we allow one of the segments to be of the form (2), but beyond this exclude the occurrence of 2's. Applying (19.2) and (18.1) to the $2 m-1$ periodical segments of the permutation $X_{\min }^{*}$ (cf. (2)) of the sequence $\left((A)_{p}\right)$, we obtain

$$
\left(Q^{\cdot}\left(X_{\min }^{\cdot}\right)\right)^{1 / p}=\dot{\alpha}_{1} \cdots \dot{\alpha}_{m}(1+o(1)) \quad(p \rightarrow \infty) .
$$

On the other hand, by (16.1) and (18.1),

$$
\left(Q^{\cdot}\left((A)_{p}\right)\right)^{1 / p}=\llbracket \dot{q}\left(a_{1}, \ldots, a_{m}\right) \rrbracket^{\cdot}(1+o(1)) \quad(p \rightarrow \infty) .
$$

Now (17.1) follows on combining this with $Q^{\cdot}\left(X_{\min }^{\cdot}\right) \leqslant Q^{\bullet}((A))$. If $a_{1}=\cdots=a_{m}$ $>2$, then equality in (17.1) follows from the same relations and the trivial equality $X_{\text {min }}^{\cdot}=\left((A)_{p}\right)$; and if $a_{1}=\cdots=a_{m}=2$, then $\llbracket \dot{q}\left((2)_{m}\right) \rrbracket^{\circ}=\llbracket 2 \rrbracket^{\circ}=1=\llbracket a_{i} \rrbracket^{\circ}$. We proceed similarly with (17.2) to complete the proof of Theorem 4. 
7. Proof of the Corollary. Applying (19.1) to the $2 r-1$ periodical segments of $X_{\max }$ (cf. (3)) and using (18.2) we obtain

$$
\log \left(Q\left(X_{\max }\right)\right)^{1 / n}=\sum_{k=1}^{r} \frac{p_{k}}{n} \log \llbracket b_{k} \rrbracket+O\left(\frac{1}{n}\right) \quad(n \rightarrow \infty) .
$$

As we remarked earlier (see (10)), $X_{\min }$ also consists of at most $2 r-1$ periodical segments (with periods $\left.\left(b_{k_{1}} b_{j_{1}}\right)\right)$. For $m=2, A=(a, b)$, the identity (16.2) reads

$$
Q\left((a b)_{p}\right)=Q^{\cdot}\left((q)_{p}\right)-Q \cdot\left((q)_{p-1}\right), \quad q=a b+2 .
$$

With the notation of (10) it follows from (18.1) and (19.1) that

$$
\log \left(Q\left(X_{\min }\right)\right)^{1 / n}=\sum_{i} \frac{m_{i}}{n} \log \llbracket b_{k_{i}} \cdot b_{j_{i}}+2 \rrbracket^{\cdot}+O\left(\frac{1}{n}\right) \quad(n \rightarrow \infty) .
$$

Both $O$-terms depend on $r$. From (20) we obtain (5.2) by some simple manipulations. Note that

$$
\llbracket a_{1} \rrbracket \llbracket a_{2} \rrbracket / \llbracket a_{1} a_{2}+2 \rrbracket \geqslant 1
$$

with equality exactly if $a_{1}=a_{2}$ (cf. (17.2) with $m=2$ ). This shows that $\lim$ $\inf \rho(P)=0$ if $\lim \sup p_{k} / n=1$ for some $k$ (and so $\lim \inf p_{i} / n=0$ for $i \neq k$ ), since there will be blocks of $b_{k}$ 's in both $X_{\min }$ and $X_{\max }$, with ratios $l_{k} / n$ of the block lengths arbitrarily close to 1 . Conversely, if $0<c<p_{k} / n<c^{\prime}<1$ for some $k$, then (5.1) clearly shows $\rho(P)>$ const $(>0)$. Similarly the behaviour of $\dot{\rho}(P)$ is analyzed. Finally, we observe that for fixed $a_{1}\left(<a_{2}\right)$ the ratio (21) is increasing with $a_{2}$ to the limit $\llbracket a_{1} \rrbracket / a_{1}$, which is in turn $\leqslant \llbracket 1 \rrbracket=(1+\sqrt{5}) / 2$ with equality iff $a_{1}=1$. From (5.1) it follows that it suffices to consider sequences with $b_{1}=1$ and large $b_{2}$. But then for given $B=\left\{1, b_{2}\right\}$ and $n \in \mathbf{N}, \sigma(p, n-p)$ is maximal when $p=[n / 2]$, and $\sigma(p, p)=\sigma(p, p+1)$ is increasing to the limit $\frac{1}{2} \log \llbracket 1 \rrbracket$ as $b_{2} \rightarrow \infty$. This proves (5.3).

8. The relations (8) and (16.3) suggest a more general class of extremum problems: let a matrix $A=\left(a_{i k}\right)$ be given with some of the entries fixed, the other elements being subject to a permutation group. One may ask for the arrangement minimizing or maximizing a certain real matrix-function. We mention two examples.

(1) Is it true that $\operatorname{Per}\left(a_{i k}\right)$, with $a_{11} \leqslant a_{12} \leqslant \cdots \leqslant a_{1 n} \leqslant a_{21} \leqslant \cdots \leqslant a_{2 n} \leqslant \cdots \leqslant$ $a_{n n}$, minimizes $\operatorname{Per}\left(x_{i k}\right)$, with $\left(x_{11} \cdots x_{n n}\right)$ varying over all permutations of $\left(a_{11}\right.$ $\cdots a_{n n}$ )? (For $n=2$ this is trivial.) Is the arrangement unique up to the obvious possibilities of interchanging equal elements and permuting rows and columns? What about $\left|\operatorname{Det}\left(x_{i k}\right)\right|$ and the maxima for both functions?

(2) As stated in [10], the extremal arrangements (2), (3) are the same for cyclic and ordinary continuants (cf. (16.3) and (8)) if the entries are all distinct. Is there an analogue to Theorem 1 in the general case?

ACKNOWLEDGEMENT. This paper was written during a stay at the University of Erlangen-Nürnberg. I should like to express miy deep gratitude to Professor K. Strambach for this opportunity and the valuable help he gave me during the work. 


\section{REFERENCES}

1. I. Borosh, Rational continued fractions with small partial quotients, Notices Amer. Math. Soc. 23 (1976), A-52, Abstract 731-10-29.

2. T. W. Cusick, The connection between the Lagrange and Markov spectra, Duke Math. J. 42 (1975), 507-517.

3. Continuants with bounded digits, Mathematika 24 (1977), 166-172.

4. L. E. Dickson, Introduction to the theory of numbers, Univ. of Chicago Press, Chicago, 1930.

5. V. Jarnik, Zur metrischen Theorie der diophantischen Approximationen, Prace Mat. Fiz. 36 (1928), $91-106$.

6. I. J. Good, The fractional dimension theory of continued fractions, Proc. Cambridge Philos. Soc. 37 (1941), 199-228.

7. E. Hlawka, Das Werk Perrons auf dem Gebiet der diophantischen Approximationen, Jber. Deutsch. Math.-Verein. 80 (1978), 1-12.

8. R. Kaufman, Continued fractions and Fourier transforms, Mathematika 27 (1980), 262-267.

9. C. G. Lekkerkerker, Geometry of numbers, Wolters-Nordhoff, Groningen; North-Holland, Amsterdam, 1969.

10. T. S. Motzkin and E. G. Straus, Some combinatorial extremum problems, Proc. Amer. Math. Soc. 7 (1956), 1014-1021.

11. G. Ramharter, Üher asymmetrische diophantische Approximationen, J. Number Theory 14 (1982), 269-279.

12. S__ Some metrical properties of continued fractions, Mathematika (to appear).

13. C. A. Rogers, Some sets of continued fractions, Proc. London Math. Soc. (3) 14 (1964), 29-44.

14. F. Supnick, On the dense packing of spheres, Trans. Amer. Math. Soc. 65 (1949), 14-26.

15. __ Optimal closed paths through certain point sets, Bull. Amer. Math. Soc. Abstract 62-1-34.

Institut fuer ANalysis, Techn. Universitaet Wien, A-1040 Gusshausstrasse 27, Austria 